\title{
Concentration and pH effect on the electronic circular dichroism and anisotropy spectra of aqueous solutions of glyceric acid calcium salt
}

\author{
Jana Bocková, ${ }^{[a]}$ Nykola C. Jones, ${ }^{[b]}$ Vanessa Leyva, ${ }^{[a]}$ Marc Gaysinski, ${ }^{[a]}$ Søren V. Hoffmann, ${ }^{[b]}$ \\ and Cornelia Meinert*[a]
}

\begin{abstract}
Electronic circular dichroism (ECD) and anisotropy spectra carry information on differential absorption of leftand right-circularly polarised light (LCPL and RCPL) by optically active compounds. This makes them powerful tools for the rapid determination of enantiomeric excesses $(e e)$ in asymmetric synthetic and pharmaceutical chemistry, as well as for predicting the ee inducible by UV CPL. The ECD response of a chiral molecule is, however, critically dependent on the properties of the surrounding medium. Here, we report on the first ECD/anisotropy spectra of aqueous solutions of the calcium salt dihydrate of glyceric acid. A systematic study of the effect of the salt concentration and $\mathrm{pH}$ on the chiroptical response revealed significant changes and the appearance of a new ECD band of opposite sign. Based on the literature this can be rationalised by the increase in the relative proportion of free glyceric acid/glycerate to $\mathrm{Ca}^{2+}$ complexes with glycerate with decreasing salt concentration or $\mathrm{pH}$. Glyceric acid can be readily produced under astrophysical conditions. The anisotropy spectra of the solution containing prevalently the free form of this dihydroxy carboxylic acid resemble the ones of previously investigated aliphatic chain hydroxycarboxylic acids and proteinogenic amino acids. This indicates possible common handedness of stellar CPL-induced asymmetry in the potential co-monomers of primitive proto-peptides.
\end{abstract}

Keywords: circularly polarised light, chiral hydroxycarboxylic acid, enantiomeric excess, complexation, alkaline earth metal, homochirality, CPL scenario.

\section{Introduction}

Differentiation between enantiomers of a chiral species requires a chiral force. Circularly polarised light (CPL) represents a true chiral entity ${ }^{1}$ and the origin of biological homochirality - uniformity of handedness of amino acids (L-) and sugars (D-) in the biosphere - remains, up to now, most likely linked to the asymmetric interaction of stellar UV CPL with enantiomers of chiral molecules. ${ }^{2,3}$ The intrinsic response of an optically active molecule to UV CPL is polarisation and wavelength dependent. This dependence is manifested in electronic circular dichroism (ECD) spectroscopy. ECD measures the differential absorption of leftand right-CPL (LCPL and RCPL) as a function of wavelength, $\Delta \varepsilon=\varepsilon_{\mathrm{L}}-\varepsilon_{\mathrm{R}}$, where $\varepsilon_{\mathrm{L}}$ and $\varepsilon_{\mathrm{R}}$ are extinction coefficients of LCPL and RCPL, respectively. The measure of the effectiveness of an asymmetric photolysis of a racemic mixture by $\mathrm{CPL}$ is given by the anisotropy factor $g=\frac{\Delta \varepsilon}{\varepsilon}$, where $\varepsilon=\frac{\varepsilon_{L}+\varepsilon_{R}}{2}$ is the extinction coefficient. The magnitude of the ee inducible by CPL $\left(e e_{L}=\frac{[L]-[D]}{[L]+[D]}\right.$, where $[L]$ and $[D]$ are concentrations of $L-$ and D-enantiomers, respectively) can be approximated as follows ${ }^{4,5}$

$|\% e e| \geq\left(1-(1-\xi)^{\frac{|g|}{2}}\right) \times 100 \%$

where $\xi$ is the extent of a photochemical reaction and the sign of $\% e e$ follows the sign of $g$. Apart from elucidating the ee inducible by $C P L, E C D$ is widely exploited to discern the enantiopurity of chiral pharmaceuticals, agrochemicals, and specialty chemicals in the fields of asymmetric synthesis and catalysis. ${ }^{6-9}$

Importantly, electronic circular dichroism is critically dependent on the conformation of the molecule as well as on the properties of the surrounding medium. ${ }^{10-12}$ The effect of $\mathrm{pH}$, temperature and solvent polarizability on the ECD of chiral amino acids in the liquid phase has been well addressed in the litearture..$^{11,13-17}$ It should be noted that varying any of the above-mentioned parameters has been found to only induce shifts in the position and intensity of the UV ECD bands. This implies that although the magnitude of the ee inducible by interaction with broadband CPL would depend on the exact conditions, the sign of the ee in such cases remains the same.

Significant changes in the ECD spectra of amino ${ }^{18-22}$ and hydroxycarboxylic acid ( $\mathrm{HCA})^{23}$ solutions were observed in the presence of transition metal ions as a result of complexation. In addition to structural changes (chelate ring formation), coordination of an optically active molecule (ligand) to a transition metal ion induces rotational strength into its $d$-d electronic transitions which gives rise to new ECD signals. ${ }^{18-22}$ This phenomenon has been recently exploited in a novel chiroptical ee sensing technique which is based on the detection of induced visible ECD bands by complexation of transition metal ions with chiral organic analytes. ${ }^{24,25}$ In addition to transition metal ions, it has also been shown that alkali $\left(\mathrm{Li}^{+}, \mathrm{Na}^{+}, \mathrm{K}^{+}\right)$and alkaline earth metal ions $\left(\mathrm{Mg}^{2+}, \mathrm{Ca}^{2+}, \mathrm{Sr}^{2+}, \mathrm{Ba}^{2+}\right)^{26-33}$ have a tendency to form complexes with hydroxycarboxylates. Their presence in solutions of HCAs was shown to induce changes in the intensity and

[a] Dr J. Bocková, V. Leyva, Dr M. Gaysinski, Dr C. Meinert Institut de Chimie de Nice, CNRS UMR 7272 Université Côte d'Azur

Avenue Valrose 28, 06 108, Nice, France

E-mail:cornelia.meinert@univ-cotedazur.fr

[b] $\operatorname{Dr}$ N. C. Jones, Dr S. V. Hoffmann

ISA, Department of Physics and Astronomy Aarhus University

8000 Aarhus C, Denmark

Received: ((will be filled in by the editorial staff))

Revised: ((will be filled in by the editorial staff)) Published online: ((will be filled in by the editorial staff)) 
position of the ECD bands extrema corresponding to the $n \pi^{*}$ transition of the carbonyl chromophore. ${ }^{29}$ The differences in the ECD signal were found to be critically dependent on (1) the nature of the ionic medium ${ }^{29,34}$ confirming the formation of complexes, ${ }^{29}$ (2) the type of the ligand which was ascribed to the extent of conformational modification of anions upon complexation, ${ }^{29}$ and (3) the ionic strength, where the higher ionic strength was linked to the higher extent of complexation. ${ }^{29}$ In addition to the change of the relative intensity of the negative ECD band of L-tartaric acid in aqueous solution at around $210-215 \mathrm{~nm}$, Hoffmann et al. ${ }^{34}$ reported a gradual increase of a newly appearing negative ECD band at around $193 \mathrm{~nm}$ upon addition of $\mathrm{NaOH}$. Based on density functional theory (DFT) calculations, this effect was assigned to the change in the preferred structural symmetry between fully protonated tartaric acid and completely ionised tartrate in the presence of metal ions. ${ }^{34}$

Interestingly, our recent study ${ }^{35}$ has shown that the ECD/anisotropy spectra of aqueous solutions of aliphatic chain HCAs resemble those of proteinogenic amino acids, suggesting that broadband stellar CPL has the potential to induce a net ee of the same handedness in the two families of chiral molecules. This has strong implications in the origin-of-life research, since these species have been discussed as the most likely monomers of primitive proto-peptides preceding contemporary peptides. ${ }^{36-38}$ However, some enantiomerically pure standards of prebiotically relevant species are commercially available only in the form of salts of alkali/alkaline earth metal ions (e.g. glyceric acid, gluconic acid, glycerol-1/3-phosphate) due to their low stability in the solidphase. ${ }^{39}$ Here we report on the first ECD/anisotropy spectroscopy experiments on aqueous solutions of the calcium salt of glyceric acid, which is a chiral dihydroxy carboxylic acid (Figure 1 a). Glyceric acid was found in UV-irradiated interstellar/cometary ice analogues (1:1 mixture of $\left.\mathrm{CH}_{3} \mathrm{OH}: \mathrm{NH}_{3}, 80 \mathrm{~K}\right)^{40}$ as well as in meteoritic samples. ${ }^{41,42}$ This suggests the molecule was very likely present on the early Earth and could have been involved in co-building proto-peptides. ${ }^{36-38}$ The present ECD/anisotropy spectra of the aqueous solutions of glyceric acid calcium salt dihydrate revealed significant changes as a function of concentration and $\mathrm{pH}$, notably the appearance of a new band of opposite sign as well as changes in the intensity and position of the extrema of the bands. The present study, therefore, highlights the importance of carefully monitoring the equilibrium concentration and acidity of solutions of alkaline earth metal salts in ECD/anisotropy studies aimed at investigating enantiopurity, as well as elucidating CPL inducible enantiomeric excesses of astrochemically relevant chiral molecules in their free form, in which they would most likely be found in interstellar environments. Comparison of the anisotropy spectrum of the free acid with those of previously investigated proteinogenic amino acids ${ }^{11,13}$ and $\mathrm{HCAs}^{35}$ brings additional insights into potential common CPL inducible ee in candidate ancestral co-building blocks of protopeptides.

\section{Materials and Methods}

Enantiopure standards of D- and L-enantiomers of glyceric acid calcium salt dihydrate (Figure 1 a) were purchased from SigmaAldrich (purity $99 \%$ and $\geq 97 \%$, respectively) and were used without further purification. The aqueous solutions for the ECD/anisotropy spectroscopy measurements were prepared in deionised, filtered, and UV-irradiated ultrapure water at concentrations of 100,20 , and $5 \mathrm{~g} \mathrm{~L}^{-1}$. Their $\mathrm{pH}$ was measured using a pH meter (InoLab pH 730). The acidic solutions at pH 2 to 3.8 were adjusted by adding a $6 \mathrm{M}$ hydrochloric acid solution (diluted from a $37 \%-12 \mathrm{M}$ - solution of $\mathrm{HCl}$, Sigma-Aldrich). The rather low volumes of the acidic sample solutions prevented using a $\mathrm{pH}$ meter and their $\mathrm{pH}$ was determined with MColorpHast $\mathrm{pH}$ indicator strips (non-bleeding, range 2-9).

For ECD/anisotropy spectroscopy experiments on free glyceric acid/glycerate, $1.444 \mathrm{~g}$ of glyceric acid calcium salt was dissolved in $12 \mathrm{ml}$ of $\mathrm{D}_{2} \mathrm{O}$ and passed, sequentially, through two cation exchange columns each containing $5 \mathrm{~g}$ of BT AG $50 \mathrm{~W}-\mathrm{X} 8$ (100200 mesh) resin. The columns had been equilibrated and washed with four bed volumes (BV) of $1 \mathrm{M} \mathrm{HCl}$, followed by four BV of MilliQ ${ }^{\circledR}$ water, and $1.5 \mathrm{BV}$ of $\mathrm{D}_{2} \mathrm{O}$. The eluted solution from the first column, employing four $\mathrm{BV}$ of $\mathrm{D}_{2} \mathrm{O}$, was preconcentrated to $15 \mathrm{ml}$ and passed through the second column using four $\mathrm{BV}$ of $\mathrm{D}_{2} \mathrm{O}$. Finally, the second solution was preconcentrated to a final volume of $12 \mathrm{ml}$. The approximate concentration of glyceric acid in the purified solution, as determined by ${ }^{1} \mathrm{H}$ NMR, was $0.75 \mathrm{M}$. The $\mathrm{pH}$ of the solution was determined to be 1-1.5 with MQuant $\mathrm{pH}$ indicator strips (non-bleeding, range 0-6).

Electronic circular dichroism and anisotropy spectra of D- and Lenantiomers of glyceric acid calcium salt dihydrate were recorded in the 170-280 $\mathrm{nm}$ wavelength range using the synchrotron radiation electronic circular dichroism spectrophotometer on the AU-CD beamline at the ASTRID2 synchrotron storage ring facility, Aarhus University, Denmark. The technique described in detail in Evans et al. ${ }^{43}$ allowed the simultaneous measurement of extinction $(\varepsilon)$ and differential extinction $(\Delta \varepsilon)$ coefficients. The spectra of the aqueous sample solutions were measured at 24.2 $\pm 0.5^{\circ} \mathrm{C}$ using quartz cells with nominal path lengths of $2,10,100$ and $500 \mu \mathrm{m}$. All spectra were recorded with a $2 \mathrm{~s}$ dwell time per point, $1 \mathrm{~nm}$ steps and multiple accumulations for each sample. The ECD spectra were mildly smoothed with a 7-point SavitzkyGolay filter.

a)

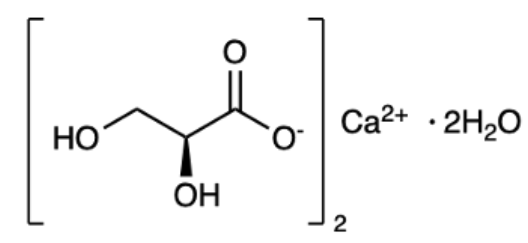

L-glyceric acid calcium salt dihydrate

b)

$$
\begin{aligned}
& \mathrm{CaGla}_{2}(\mathrm{~s}) \rightleftharpoons \mathrm{CaGla}^{+}+\mathrm{Gla}^{-} \\
& \mathrm{CaGla}^{+} \rightleftharpoons \mathrm{Ca}^{2+}+\mathrm{Gla}^{-}
\end{aligned}
$$

FIGURE 1 a) Chemical formula of the L-enantiomer of glyceric acid calcium salt dihydrate and b) schematic of stepwise dissolution of calcium salt of glyceric acid $\mathrm{CaGla}_{2}(\mathrm{~s})$, where $\mathrm{CaGla}+$ is $1: 1$ complex of glycerate Gla and calcium ion $\mathrm{Ca}^{2+}$.

\section{Results and Discussion}

The electronic circular dichroism spectra of aqueous solutions of D- and L-enantiomers of glyceric acid calcium salt dihydrate measured at $24.2 \pm 0.5^{\circ} \mathrm{C}$ at three different concentrations are shown in Figure $\mathbf{2}$ a to $\mathbf{c}$ along with their corresponding anisotropy spectra in Figure $2 \mathbf{d}$ to $\mathbf{f}$. The ECD spectra significantly differ from each other, which suggests that different structures 
contribute to the ECD signals in Figure $\mathbf{2}$ a to $\mathbf{c}$. The reliability of the recorded data is confirmed by the mirror symmetry of the ECD signal of the two enantiomers at each concentration (nearly equal magnitude and position of extrema). Given the uncertainties induced by low absorbance and ECD signals above $\sim 240$ $250 \mathrm{~nm}$, the values of the anisotropy factor $g$ are less reliable in this wavelength range as indicated by dashed line in Figure $\mathbf{2} \mathbf{d}$ to $\mathrm{f}$.

Dissolution of calcium salt of glyceric acid in water is a two-step equilibrium process and it is schematically shown in Figure 1 b. ${ }^{31,44}$ Calcium salts of hydroxycarboxylic acids were found to be

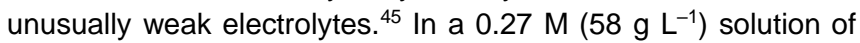
calcium lactate and $0.08 \mathrm{M}\left(32.7 \mathrm{~g} \mathrm{~L}^{-1}\right)$ solution of calcium gluconate at $20^{\circ} \mathrm{C}$ only about $21 \%$ and $30 \%$ of calcium, respectively, is present in the free form. ${ }^{31}$ Glyceric acid is an aliphatic polyhydroxy carboxylic acid with two hydroxyl groups on the $\mathrm{C}_{\alpha^{-}}$and $\mathrm{C}_{\beta}$ - atoms (Figure 1 a). Several experimental studies have confirmed that the carboxylate group (COO-) is capable of coordinating to alkali $\left(\mathrm{Li}^{+}, \mathrm{Na}^{+}, \mathrm{K}^{+}\right)$/alkaline earth metal ions $\left(\mathrm{Mg}^{2+}\right.$, $\left.\mathrm{Ca}^{2+}, \mathrm{Sr}^{2+}, \mathrm{Ba}^{2+}\right) .{ }^{26-33}$ Moreover, ${ }^{13} \mathrm{C}$ NMR experiments, ${ }^{27,28,30}{ }^{1} \mathrm{H}-$ ${ }^{43} \mathrm{Ca}$ NMR experiments ${ }^{32,33}$ as well DFT and Hartree-Fock ab initio calculations ${ }^{31} 32,33$ showed that in addition to the carboxylate group, a hydroxyl group can be involved in such coordination in the presence of a negatively charged group nearby (e.g. COO'). Pallagi et al. $^{32}$ reported that gluconate - another aliphatic polyhydroxy carboxylic acid - acts as a multidentate ligand in aqueous solution with $\mathrm{Ca}^{2+}$, with the binding sites being the oxygens of the carboxylate and hydroxyl groups on the $C_{\alpha}, C_{\beta}$, and $\mathrm{C}_{\varepsilon}$. On the other hand, Vavrusova et al. ${ }^{31}$ reported a coexistence of two conformers in a solution of calcium gluconate: (1) the conformation with $\mathrm{Ca}^{2+}$ bound to two carboxylate oxygens and the oxygen of the hydroxyl group on the $C_{\beta}$, as well as (2) the conformation where $\mathrm{Ca}^{2+}$ binds to only one oxygen of the carboxylate group and to the oxygens of the hydroxyl groups on the $C_{\alpha}$ and $C_{\beta}$. Based on the strong precedent in the literature for the interaction of $\mathrm{Ca}^{2+}$ with the carboxylate ${ }^{26-33}$ and hydroxy $\left.\right|^{27,28,30-33}$ groups of hydroxycarboxylates, we therefore attribute part of the spectral changes in our ECD data to such ionselective pairing. The precise conformations have yet to be determined with the help of theoretical calculations and/or ${ }^{1} \mathrm{H}-$ ${ }^{43} \mathrm{Ca}$ NMR spectroscopy experiments.

With decreasing glyceric acid calcium salt dihydrate concentration, we expect a decrease in the extent of complexation, i.e. lower relative abundance of complexes of glycerate and $\mathrm{Ca}^{2+}$ to free glyceric acid/glycerate (at constant $\mathrm{K}_{\mathrm{a}}$ ). This could explain the gradual shift of the negative ECD band to shorter wavelengths in the solution of L-glyceric acid calcium salt dihydrate from $100 \mathrm{~g} \mathrm{~L}^{-}$ 1 to $5 \mathrm{~g} \mathrm{~L}^{-1}$ which is at least partly driven by the gradual appearance of a positive band at slightly longer wavelength (Figure $\mathbf{2}$ a to $\mathbf{c}$ and Figure $\mathbf{3}$ a to $\mathbf{c}$ ). It should be noted that the $\mathrm{pH}$ of the three solutions $\left(100,20\right.$, and $\left.5 \mathrm{~g} \mathrm{~L}^{-1}\right)$ did not vary significantly (Figure $\mathbf{3}$ a to $\mathbf{c}$ ). This picture is in line with the ECD spectra of L-glyceric acid calcium salt dihydrate (Figure $\mathbf{3} \mathbf{b}$ and d to $\mathbf{f}$ ) showing further increase in the intensity of the longer wavelength positive ECD band upon addition of $6 \mathrm{M} \mathrm{HCl}$. $\mathrm{HCl}$ is a strong acid, addition of which results in the dissociation of ion complexes and protonation of glycerate. In addition, some formation of ion pairs such as $\mathrm{CaCl}^{+}$can be expected ${ }^{46}$. At pH $\sim 2$ 2.5 we expect the majority of glyceric acid to be present in its free form (Figure $3 \mathbf{f}$ ), i.e. without bound $\mathrm{Ca}^{2+}$. The anisotropy spectra of 5,20 and $100 \mathrm{~g} \mathrm{~L}^{-1}$, with the $\mathrm{pH}$ adjusted using $6 \mathrm{M} \mathrm{HCl}$ to $\sim 2$ 2.5 , all show a positive band with the maximum at around $210 \mathrm{~nm}$ (Figure 4). Moreover, they resemble well the anisotropy spectrum of the L-glyceric acid calcium salt dihydrate solution after the removal of $\mathrm{Ca}^{2+}$ by cation exchange chromatography (Figure 4). Hence the anisotropy band at around $210 \mathrm{~nm}$ can be undoubtedly ascribed to pure glyceric acid/glycerate. The intensity of this band in the solutions at $\mathrm{pH} \sim 2-2.5$ is slightly lower at $100 \mathrm{~g} \mathrm{~L}^{-}$ ${ }^{1}$ compared to the two lower concentrations. This could be explained by a slightly higher net $\mathrm{pH}$ of this solution that was not measurable within the uncertainty of the $\mathrm{pH}$ indicator strips. However, it is clearly visible in Figure $\mathbf{3} \mathbf{j}$ to $\mathbf{I}$ that with decreasing $\mathrm{pH}$ the intensity of this anisotropy band increases. 
Chirality
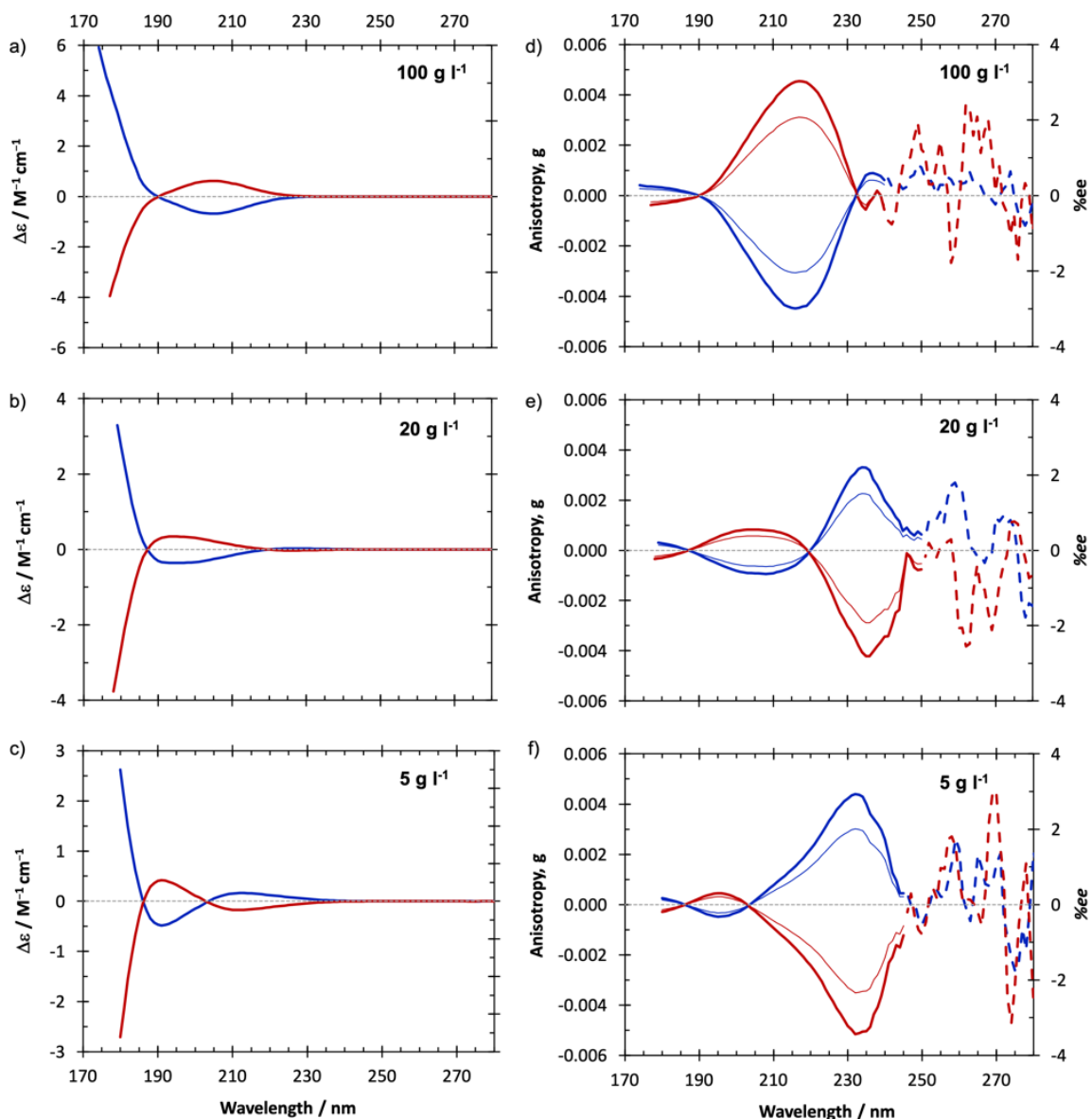

FIGURE 2 a) to c) ECD and d) to $\mathbf{g}$ ) anisotropy spectra (thick lines) of aqueous solutions of D- $(R$-, red) and L-enantiomers ( $S$-, blue) of glyceric acid calcium salt dihydrate at concentrations of 100,20 and $5 \mathrm{~g} \mathrm{~L}^{-1}$, respectively. Thin lines in d) to g) represent the lower limit of the enantiomeric excess (\%ee) inducible by either left- or right-circularly polarised light as a function of wavelength at the extent of reaction $\xi=0.9999$ calculated based on relation $(1)^{5}$. 

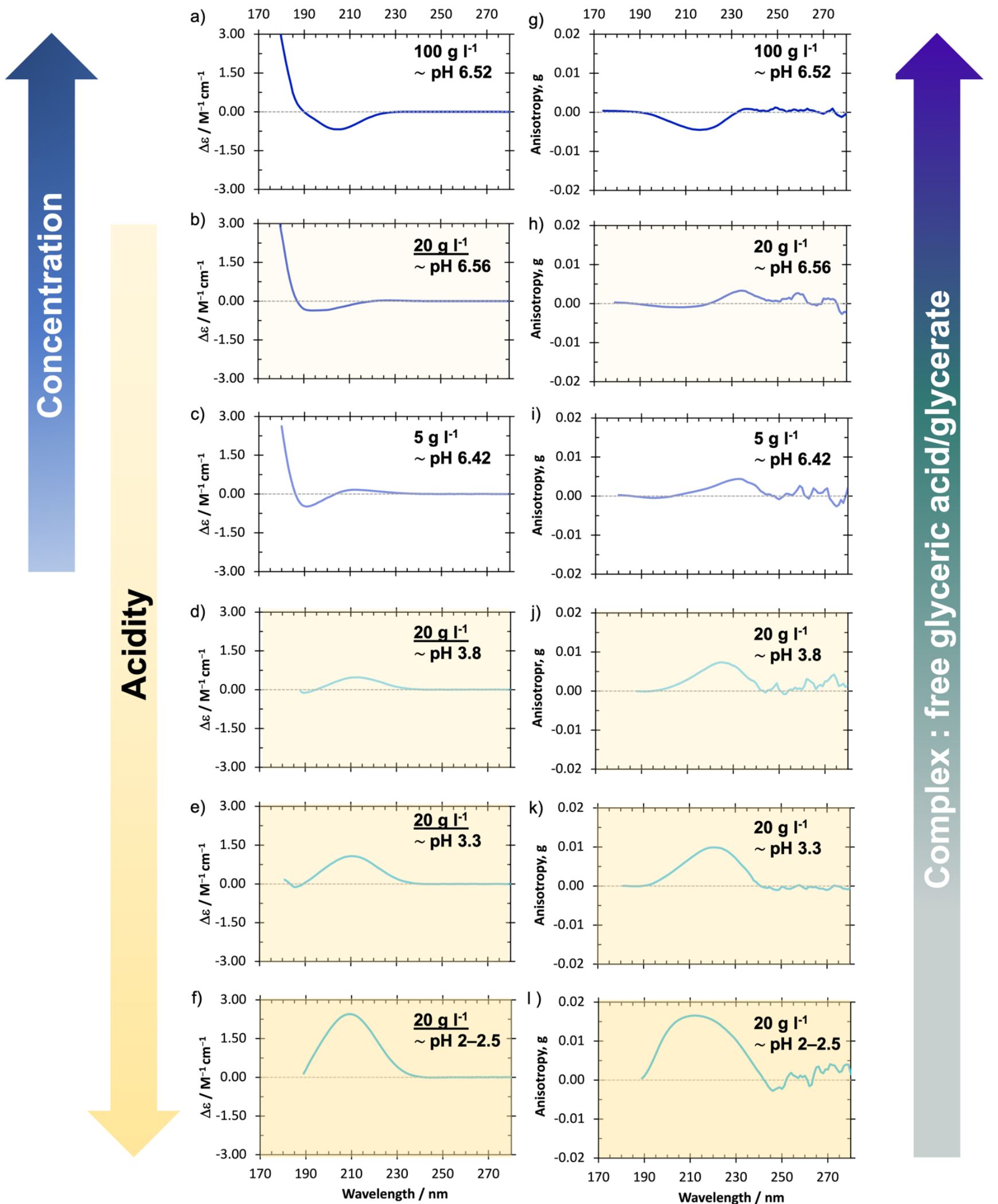

FIGURE 3 a) to c) ECD and g) to i) anisotropy spectra of aqueous solutions of L-glyceric acid calcium salt dihydrate at concentrations $100 \mathrm{~g} \mathrm{~L}^{-1}$ ( pH 6.52), $20 \mathrm{~g} \mathrm{~L}^{-1}$ ( pH 6.56) and $5 \mathrm{~g} \mathrm{~L}^{-1}$ ( pH 6.42), respectively. d) to f) ECD and j) to I) anisotropy spectra of aqueous solutions of $\mathrm{L}-\mathrm{glyceric}$ acid calcium salt dihydrate at $20 \mathrm{~g} \mathrm{~L}^{-1}$ with $\mathrm{pH}$ adjusted by $6 \mathrm{M} \mathrm{HCl}$ to $\sim 3.8, \sim 3.3$ and $\sim 2-2.5$, respectively. The blue arrow indicates increase in the concentration for solutions at similar $\mathrm{pH}$. The yellow arrow indicates increase in the acidity of the solutions with concentration $20 \mathrm{~g} \mathrm{~L}^{-1}$ (corresponding spectra with yellow gradient). The arrow on the right indicates expected increase in the extent of complexation. 
The dominant anisotropy spectral band of free L-glyceric acid in the 170-280 nm wavelength range is of the same sign as those of previously investigated L-HCAs ${ }^{35}$ (aliphatic chain, malic, and mandelic acid) and proteinogenic L-amino acids ${ }^{11,13}$. This suggests that $\mathrm{CPL}$ acting in this wavelength range, which is the dominant UV emission wavelength range of the majority of normal stars, ${ }^{35,47,48}$ can induce an enantiomeric excess of the same handedness in these molecular species, which were likely present on the early Earth ${ }^{40-42}$ and thought to be involved in co-building primitive proto-peptides. ${ }^{36-38}$ Cooper and Rios ${ }^{42}$ reported racemic or near racemic glyceric acid concentrations in carbonaceous chondrites. However, some of the analyses were accompanied by poor enantioseparation, potential contamination could not be fully excluded, and the analytical uncertainties were not stated. Based on the anisotropy spectra in Figure 5 and relation (1) the maximum enantiomeric excess of $\geq 7.6 \%$ could be induced by an asymmetric photolysis by RCPL at $210 \mathrm{~nm}$ at the extent of reaction $\xi=0.9999$. It should be noted that in the present-day biosphere, glyceric acid is primarily found in the $\mathrm{D}$-form ${ }^{49}$ mainly as an end product of metabolic pathways. ${ }^{50}$ This, however, does not necessarily reflect on its preferential handedness on the early Earth. Instead, enantioselective analyses of meteoritic samples will be invaluable for shedding light on the relevance and conditions of the CPL scenario.

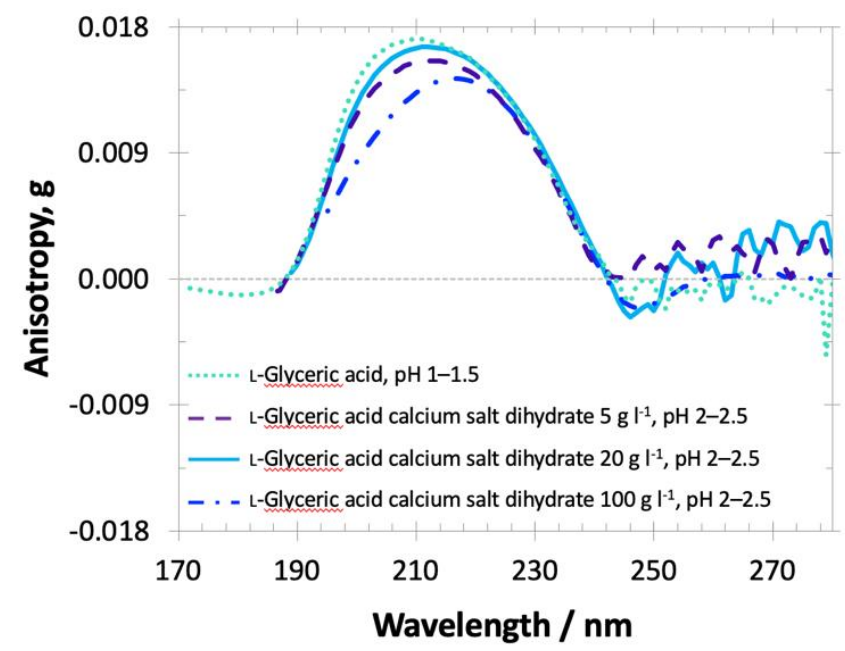

FIGURE 4 Comparison of the anisotropy spectra of aqueous solutions of L-glyceric acid calcium salt dihydrate at concentrations 5, 20 and $100 \mathrm{~g} \mathrm{~L}^{-1}$ with $\mathrm{pH}$ adjusted by $6 \mathrm{M} \mathrm{HCl}$ to $\sim 2-2.5$ with the anisotropy spectrum of free L-glyceric acid $\left(\mathrm{D}_{2} \mathrm{O}\right.$ solution of L-glyceric acid calcium salt dihydrate after purification from $\mathrm{Ca}^{2+}$ ).

The comparison of the anisotropy spectrum of free L-glyceric acid $\left(\mathrm{D}_{2} \mathrm{O}\right.$ solution of $\mathrm{L}$-glyceric acid calcium salt dihydrate after purification from $\mathrm{Ca}^{2+}$ ) with those of L-lactic acid (a representative of aliphatic chain HCAs) and L-glyceric acid's amino acid analogue L-serine is shown in Figure 5. It is reasonable to assume that the positive ECD band with the maximum at around $210 \mathrm{~nm}$ is associated with the $n \pi^{*}$ transition of the carboxyl chromophore of free glyceric acid/glycerate. On the contrary, the negative band in Figure $\mathbf{3}$ a to $\mathbf{c}$ is then most likely linked to the formation of $1: 1$ complexes of glycerate and $\mathrm{Ca}^{2+}$. The presence and hence contribution of 2:1 complexes cannot be ruled out, however, it is expected to be negligible. ${ }^{31,32}$ Theoretical calculations and/or ${ }^{1} \mathrm{H}-{ }^{43} \mathrm{Ca}$ NMR spectroscopy experiments will be necessary to confirm the contributing molecular conformations and determine the electronic excited states involved. Moreover, complexometric titration in combination with electrochemical measurement of calcium ion activity can be used to elucidate association constants for complex formation. ${ }^{31}$

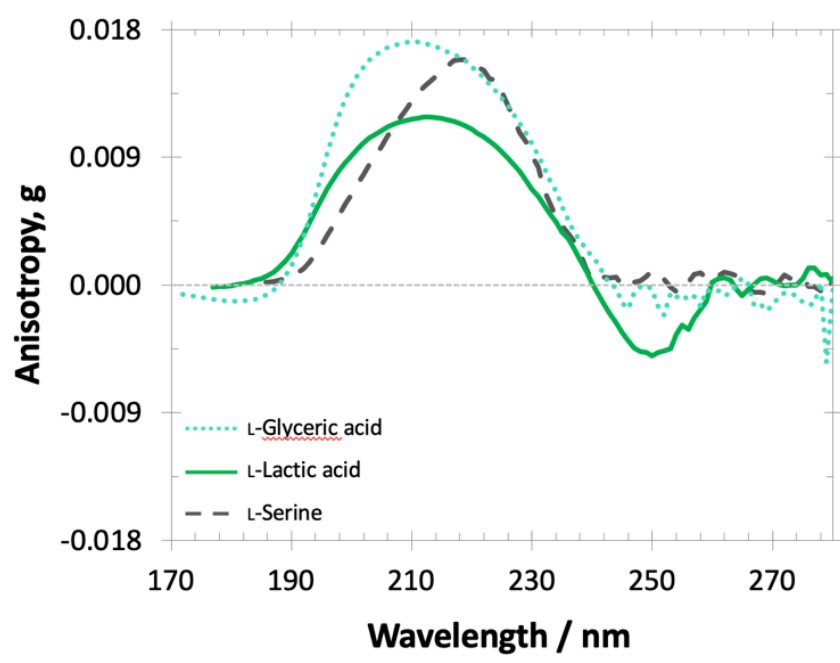

FIGURE 5 Comparison of the anisotropy spectra of aqueous solutions of the L-enantiomers of glyceric acid $\left(\mathrm{D}_{2} \mathrm{O}\right.$ solution of L-glyceric acid calcium salt dihydrate after purification to remove $\mathrm{Ca}^{2+}$ ), the $\mathrm{HCA}$ lactic acid $^{35}$ and the glyceric acids' amino acid analogue serine ${ }^{11}$ in the dominant UV emission wavelength range of the majority of normal stars $^{35,47,48}$ indicating the same handedness of stellar CPL-inducible enantiomeric excess.

\section{Conclusion}

The present paper highlights the effects of concentration and $\mathrm{pH}$ on the electronic circular dichroism and anisotropy spectra of aqueous solutions of the calcium salt of glyceric acid. Based on previous studies these can be ascribed to the changes in the extent of complexation of $\mathrm{Ca}^{2+}$ and glycerate. Concentration and acidity must therefore be carefully considered in any ECD/anisotropy spectroscopy studies aimed at investigating enantiomeric excesses inducible by CPL or the enantiopurity of a mixture. On the other hand, ECD spectroscopy can be exploited for determination of association constants.

Adjusting the $\mathrm{pH}$ of the aqueous solutions of L-glyceric acid calcium salt dihydrate to $\sim 2-2.5$ was shown to reduce the complex formation. The corresponding ECD/anisotropy spectra resemble well the anisotropy spectrum of the free L-glyceric acid derived from a L-glyceric acid calcium salt dihydrate solution after the removal of $\mathrm{Ca}^{2+}$ ions by ion exchange chromatography. The anisotropy spectrum of pure L-glyceric acid indicates the same handedness of a broadband UV (170-280nm) CPL inducible enantiomeric excess as in the previously investigated hydroxycarboxylic acids and proteinogenic amino acids, which could have been common constituents of ancestral proto-peptides.

\section{Acknowledgements}

This research has received funding from the European Research Council under the European Union's Horizon 2020 research and innovation programme [grant agreement 804144]. Further funding was provided by the French government through the UCA JEDI Investments in the Future project managed by the National 
Research Agency (ANR) with the reference number ANR-15IDEX-01 as well as by the project CALIPSOplus under the Grant Agreement 730872 from the EU Framework Programme for Research and Innovation HORIZON 2020.

\section{REFERENCES AND NOTES}

1. Barron LD. Can a Magnetic Field Induce Absolute Asymmetric Synthesis? Science 1994;266:1491-1492.

2. Bonner WA. The origin and amplification of biomolecular chirality. Orig Life Evol Biosph 1991;21:59-111.

3. Evans AC, Meinert C, Giri C, Goesmann F, Meierhenrich UJ. Chirality, photochemistry and the detection of amino acids in interstellar ice analogues and comets. Chem Soc Rev 2012;41:5447-5458.

4. Balavoine G, Moradpour A, Kagan HB. Preparation of chiral compounds with high optical purity by irradiation with circularly polarized light, a model reaction for the prebiotic generation of optical activity. J Am Chem Soc

1974; $96: 5152-5158$

5. Meinert C, Bredehöft JH, Filippi JJ, et al. Anisotropy spectra of amino acids. Angew Chemie Int Ed 2012;51:4484-4487.

6. Chen L, Zhao Y, Gao F, Garland M. Determination of enantiomeric excess using the ultraviolet-circular dichroism and the high-performance liquid chromatography-circular dichroism methods. App/ Spectrosc 2003;57:797-804.

7. Wolf $\mathrm{C}$, Bentley KW. Chirality sensing using stereodynamic probes with distinct electronic circular dichroism output. Chem Soc Rev 2013;42:5408-5424.

8. Pilicer SL, Dragna JM, Garland A, Welch CJ, Anslyn EV, Wolf $\mathrm{C}$. High-throughput determination of enantiopurity by microplate circular dichroism. J Org Chem 2020;85:1085810864.

9. Herrera BT, Pilicer SL, Anslyn EV, Joyce LA, Wolf C. Optical Analysis of Reaction Yield and Enantiomeric Excess: A New Paradigm Ready for Prime Time. J Am Chem Soc 2018:140:10385-10401.

10. Reichardt C, Welton T. Solvents and Solvent Effects in Organic Chemistry. Fourth, updated and enlarged edition Weinheim: Wiley-VCH Verlag GmbH \& Co. KGaA; 2011. $692 \mathrm{p}$.

11. Bredehöft JH, Jones NCJ, Meinert C, Evans AC, Hoffmann SV, Meierhenrich U. Understanding photochirogenesis: solvent effects on circular dichroism and anisotropy spectroscopy. Chirality 2014;26:373-378.

12. Evans AC, Petit AS, Guillen SG, Neukirch AJ, Hoffmann SV, Jones NC. Chiroptical characterization tools for asymmetric small molecules - experimental and computational approaches for electronic circular dichroism (ECD) and anisotropy spectroscopy. RSC Adv 2021;11:1635-1643.

13. Nishino H, Kosaka A, Hembury GA, Matsushima K, Inoue $\mathrm{Y}$. The $\mathrm{pH}$ dependence of the anisotropy factors of essential amino acids. J Chem Soc Perkin Trans 2002;2:582-590.

14. Nishino H, Kosaka A, Hembury GA, et al. Absolute asymmetric photoreactions of aliphatic amino acids by circularly polarized synchrotron radiation: critically $\mathrm{pH}$ dependent photobehavior. J Am Chem Soc

2002;124:11618-11627

15. Nishino $H$, Hosaka M, Katoh M, Inoue $Y$. Photoreaction of rac-leucine in ice by circularly polarized synchrotron radiation: temperature-induced mechanism switching from Norrish type II to deamination. Chem - A Eur J 2013:19:13929-13936.

16. Amdursky N, Stevens MM. Circular dichroism of amino acids: Following the structural formation of phenylalanine. ChemPhys Chem 2015;16:2768-2774.

17. Snyder PA, Vipond PM, Johnson WC. Circular dichroism of the alkyl amino acids in the vacuum ultraviolet. Biopolymers 1973;12:975-992.

18. Hawkins CJ, Wong CL. Circular dichroism spectra of amino acid complexes. III. Copper(II) compounds. Aust J Chem 1970;23:2237-2246.

19. Haines RA, Reimer M. Circular dichroism of amino acid Sullivan EA. Conformational dissymmetry. Circular dichroism of amino acid and peptide complexes. Can J Chem 1979;57:62-66.

21. Yasui T, Hidaka J, Shimura Y. Metal complexes of amino acids. III. The circular dichroism of cobalt(III) ammine and ethylenediamine complexes with L-amino acids. Bull Chem Soc Jpn 1966;39:2417-2424.

22. Yasui T. Metal complexes of amino acids. I. The circular dichroism of copper(II) complexes with optically active amino acids. Bull Chem Soc Jpn 1965;38:1746-1749.

23. Daniele PG, Prenesti E, Ostacoli G. Ultraviolet-circular dichroism spectra for structural analysis of copper(II) complexes with aliphatic and aromatic ligands in aqueous solution. J Chem Soc Dalt Trans 1996:3269-3275.

24. De los Santos ZA, Lynch CC, Wolf C. Optical chirality sensing with an auxiliary-free earth-abundant cobalt probe. Angew Chemie Int Ed 2019;58:1198-1202.

25. Lynch CC, De Los Santos ZA, Wolf C. Chiroptical sensing of unprotected amino acids, hydroxy acids, amino alcohols, amines and carboxylic acids with metal salts. Chem Commun 2019:55:6297-6300.

26. Bazin H, Bouchu A, Descotes G, Petit-Ramel M. Comparison of calcium complexation of some carboxylic acids derived from D-glucose and D-fructose. Can J Chem 1995;73:1338-1347.

27. Kondoh A, Oi T. Interaction of alkaline earth metal ions with acetic and lactic acid in aqueous solutions studied by $13 \mathrm{C}$ NMR spectroscopy. Zeitschrift fur Naturforsch A 1997:52:351-357.

28. Dudás C, Kutus B, Böszörményi É, et al. Calcium complexing behaviour of lactate in neutral to highly alkaline medium. J Mol Struct 2019;1180:491-498.

29. Daniele PG, De Stefano C, Giuffrè O, Prenesti E, Sammartano S. Interaction of L-tartaric acid with alkaline metals and open chain polyammonium cations in aqueous solution. J Chem Soc Dalt Trans 2002:435-440.

30. Kondoh A, Oi T. Interaction of alkaline earth metal ions with carboxylic acids in aqueous solutions studied by $13 \mathrm{C}$ NMR spectroscopy. Zeitschrift fur Naturforsch A 1998;53:77-91.

31. Vavrusova M, Liang R, Skibsted LH. Thermodynamics of dissolution of calcium hydroxycarboxylates in water. J Agric Food Chem 2014;62:5675-5681.

32. Pallagi A, Sebk P, Forgó P, Jakusch T, Pálinkó I, Sipos P. Multinuclear NMR and molecular modelling investigations on the structure and equilibria of complexes that form in aqueous solutions of $\mathrm{Ca}^{2+}$ and gluconate. Carbohydr Res 2010;345:1856-1864.

33. Pallagi A, Csendes Z, Kutus B, et al. Multinuclear complex formation in aqueous solutions of $\mathrm{Ca}$ (II) and heptagluconate ions. Dalt Trans 2013;42:8460-8467.

34. Hoffmann M, Grajewski J, Gawronski J. Extending the applications of circular dichroism in structure elucidation: aqueous environment breaks the symmetry of tartrate dianion. New J Chem 2010;34:2020-2026.

35. Bocková J, Jones NC, Meierhenrich UJ, Hoffmann SV, Meinert C. Chiroptical activity of hydroxycarboxylic acids with implications for the origin of biological homochirality. Commun Chem 2021;4:86.

36. Mamajanov I, Macdonald PJ, Ying J, et al. Ester formation and hydrolysis during wet-dry cycles: Generation of farfrom-equilibrium polymers in a model prebiotic reaction. Macromolecules 2014;47:1334-1343.

37. Forsythe JG, Yu SS, Mamajanov I, et al. Ester-mediated amide bond formation driven by wet-dry cycles: a possible path to polypeptides on the prebiotic Earth. Angew Chemie Int Ed 2015:54:9871-9875.

38. Frenkel-Pinter M, Haynes JW, Martin C, et al. Selective incorporation of proteinaceous over nonproteinaceous cationic amino acids in model prebiotic oligomerization reactions. Proc Natl Acad Sci U S A 2019;116:1633816346.

39. Gilbert G, Johnson WS. Preparation of glyceric acid. United States Pat Off 1956;US-2752391A.

40. Nuevo M, Bredehöft JH, Meierhenrich UJ, d'Hendecourt L, Thiemann WHP. Urea, glycolic acid, and glycerol in an organic residue produced by ultraviolet irradiation of interstellar/pre-cometary ice analogs. Astrobiology 
2010;10:245-256.

41. Cooper G, Kimmich N, Belisle W, Sarinana J, Brabham K, Garrel L. Carbonaceous meteorites as a source of sugarrelated organic compounds for the early Earth. Nature 2001;414:879-883.

42. Cooper G, Rios AC. Enantiomer excesses of rare and common sugar derivatives in carbonaceous meteorites. Proc Natl Acad Sci U S A 2016;113:E3322-E3331.

43. Evans AC, Meinert C, Bredehöft JH, et al. Anisotropy spectra for enantiomeric differentiation of biomolecular building blocks. In: Schurig V. editor. Topics in Current Chemistry. Vol 341. Heidelberg: Springer-Verlag;2013. p 271-299.

44. Vavrusova M, Munk MB, Skibsted LH. Aqueous solubility of calcium L-lactate, calcium D-gluconate, and calcium Dlactobionate: Importance of complex formation for solubility increase by hydroxycarboxylate mixtures. J Agric Food Chem 2013;61:8207-8214.

45. Davies CW. 56. The extent of dissociation of salts in water. Part VI. Some calcium salts of organic acids. J Chem Soc
1938:277-281.

46. Gao R, van Halsema FED, Temminghoff EJM, et al. Modelling ion composition in simulated milk ultrafiltrate (SMUF) II. Influence of $\mathrm{pH}$, ionic strength and polyphosphates. Food Chem 2010;122:710-715.

47. Bailey J, Chrysostomou A, Hough JH, et al. Circular polarization in star-formation regions: Implications for biomolecular homochirality. Science 1998;281:672-674.

48. Bianchi L, Conti A, Shiao B. The ultraviolet sky: An overview from the GALEX surveys. Adv Sp Res 2014;53:900-912.

49. Glavin DP, Burton AS, Elsila JE, Aponte JC, Dworkin JP The search for chiral asymmetry as a potential biosignature in our solar system. Chem Rev 2020;120:4660-4689.

50. Lassalle L, Engilberge S, Madern D, Vauclare P, Franzetti B, Girard E. New insights into the mechanism of substrates trafficking in glyoxylate/hydroxypyruvate reductases. Sci Rep 2016;6:1-13.

\section{Graphical Abstract}

The $\mathrm{pH}$ and salt concentration induce significant changes in the chiroptical response of aqueous solutions of the calcium salt of glyceric acid. These are rationalised by changes in the relative proportion of free glyceric acid/glycerate to $\mathrm{Ca}^{2+}$ complexes. The anisotropy spectra of free glyceric acid resemble the ones of previously investigated potential co-monomers of protopeptides and indicate common handedness of circularly polarised light inducible enantiomeric excess across molecular families.

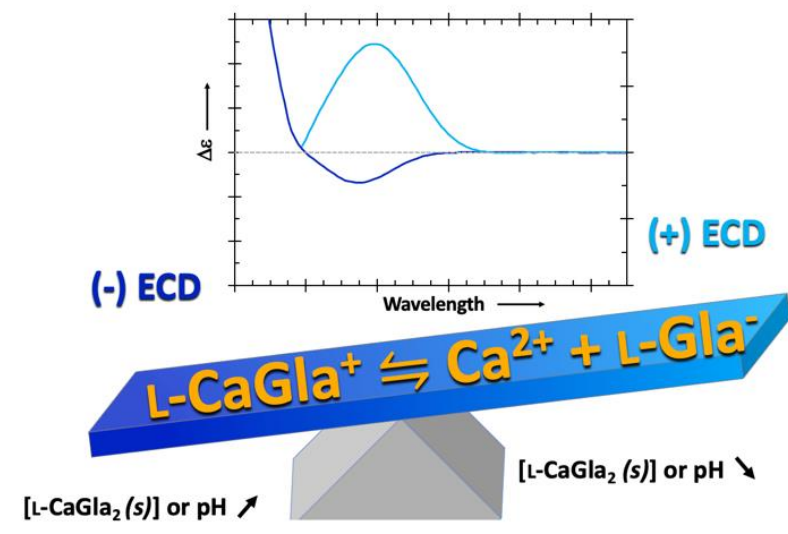

